ORIGINAL ARTICLE

\title{
Role of "Ischemia Modified Albumin", a new biochemical marker of myocardial ischaemia, in the early diagnosis of acute coronary syndromes
}

\author{
M K Sinha, D Roy, D C Gaze, P O Collinson, J-C Kaski
}

Emerg Med J 2004;21:29-34

See end of article for authors' affiliations ......................

Correspondence to: Professor J-C Kaski, Coronary Artery Disease Research Unit, St George's Hospital Medical School, Cranmer Terrace, London SW17 ORE, UK;

jkaski@sghms.ac.uk

Accepted for publication 15 September 2003

\begin{abstract}
Background: Diagnosis of cardiac ischaemia in patients attending emergency departments (ED) with symptoms of acute coronary syndromes is often difficult. Cardiac troponin (cTn) is sensitive and specific for the detection of myocardial damage but may not rise during reversible myocardial ischaemia. Ischemia Modified Albumin (IMA) has recently been shown to be a sensitive and early biochemical marker of ischaemia.

Methods and Results: This study evaluated IMA in conjunction with ECG and cTn in 208 patients presenting to the ED within three hours of acute chest pain. At presentation, a 12-lead ECG was recorded and blood taken for IMA and cardiac troponin $\mathrm{T}(\mathrm{cTnT})$. Patients underwent standardised triage, diagnostic procedures, and treatment. Results of IMA, ECG, and cTnT, alone and in combination, were correlated with final diagnoses of non-ischaemic chest pain, unstable angina, ST segment elevation, and non-ST segment elevation myocardial infarction. In the whole patient group, sensitivity of IMA at presentation for an ischaemic origin of chest pain was $82 \%$, compared with $45 \%$ of ECG and $20 \%$ of cTnT. IMA used together with cTnT or ECG, had a sensitivity of $90 \%$ and $92 \%$, respectively. All three tests combined identified $95 \%$ of patients whose chest pain was attributable to ischaemic heart disease. In patients with unstable angina, sensitivity of IMA used alone was equivalent to that of IMA and ECG combined. Conclusions: IMA is highly sensitive for the diagnosis of myocardial ischaemia in patients presenting with symptoms of acute chest pain.
\end{abstract}

A cute chest pain suggestive of unstable angina (UA) or acute myocardial infarction (AMI) accounts for about six million hospital admissions per annum in the USA. ${ }^{1}$ Only $17 \%$ of these are finally diagnosed as acute coronary syndromes (ACS $)^{2}$ caused by ischaemic heart disease (IHD). Patients with acute chest pain suggestive of ACS present with a heterogeneous array of conditions, including non-ischaemic chest pain (NICP), transient myocardial ischaemia, UA, ST segment elevation myocardial infarction (STEMI), and nonST segment elevation infarction (NSTEMI). ${ }^{3-7}$

Cardiac ischaemia is the most common mechanism underlying ACS that, when prolonged, may lead to myocardial damage and cell death. Diagnosis of ischaemia is difficult in patients presenting with acute chest pain, particularly those with uninterpretable baseline ECGs, normal ECGs during pain, or without evidence of myocardial necrosis. None of the traditional clinical variables, 12-lead ECG, biochemical markers of necrosis, and imaging techniques can be considered a true "gold standard" for the diagnosis of cardiac ischaemia. ${ }^{89}$ Although cardiac ischaemia is highly probable in the presence of ST segment or T wave changes, ${ }^{10}$ regional wall motion abnormalities ${ }^{11}$ or myocardial perfusion defects, ${ }^{12}$ are frequently absent in patients presenting to the emergency department (ED) with acute chest pain. ${ }^{13}$ Confounding factors such as early repolarisation, left ventricular hypertrophy, and left bundle branch block often preclude reliable ST segment analysis. ${ }^{14}$ Echocardiography is limited by non-ischaemic aetiologies responsible for wall motion abnormalities ${ }^{15}$ and by an injury threshold of $>20 \%$ of the myocardial wall thickness before ischaemic wall motion abnormalities can be detected. ${ }^{11}$ Sensitivity of radionuclide imaging is limited by an injury threshold of $>10 \mathrm{~g}$ of myocardium $^{16}$ and specificity is affected by attenuation artefacts. Cardiac troponins (cTn) are sensitive and specific markers of myocardial damage, but it takes about four to six hours for these biomarkers to be detectable in the circulation $^{17}$ and they do not necessarily rise in blood with reversible ischaemia.

There is a need for early and sensitive markers of cardiac ischaemia as current diagnostic tests fail to identify many chest pain patients presenting with ACS and who are at high risk for adverse cardiac events. Furthermore, the lack of sensitivity of available diagnostic tools precludes early discharge of patients without ACS. We evaluated the role of "Ischemia Modified Albumin" (IMA), a new marker of ischaemia, in patients with ACS. IMA is produced during an ischaemic attack and is present in blood in easily detectable concentrations. ${ }^{18-20}$

The aim of this study was to compare the clinical performance of IMA, cTn, and ECG, alone and in combination, in patients presenting to the ED with symptoms suggestive of acute cardiac ischaemia.

\section{METHODS}

\section{Patients}

This study was performed at St George's Hospital, London, UK, and was approved by the local ethics committee. We recruited, on a prospective sequential basis, patients who arrived at the ED within three hours of acute chest pain. The

Abbreviations: UA, unstable angina; $\mathrm{AMl}$, acute myocardial infarction; IHD, ischaemic heart disease; ACS, acute coronary syndromes; NSTEMI, non-ST segment elevation infarction; STEMI, ST segment elevation myocardial infarction; IMA, Ischemia modified albumin; ED, emergency department; CTtn, cardiac troponins; NICP, non-ischaemic chest pain; NPV, negative predictive value; PPV, positive predictive value 
enrolment period was between June and December 2001. All enrolled patients had an ECG and blood collected within two hours of arrival, and received routine institutional care blinded to the IMA results. IMA (Ischemia Technologies, Denver, Colorado, USA) and troponin $\mathrm{T}$ ( $\mathrm{CTnT}$ ) (Roche Diagnostics, Lewes, Sussex, UK) testing was performed before any heparin/thrombolytic treatment was started. Standardised clinical data were collected for each patient, which included time of presentation at the ED, approximate duration of symptoms and, details of the final discharge diagnosis. Pregnant women, patients with symptoms and signs suggestive of acute mesenteric ischaemia, acute renal failure, peripheral vascular disease, or brain ischaemia were not enrolled in the study.

A total of 245 patients were recruited for the study. However, 26 patients were excluded from the final analysis as a result of inaccuracy of timing for sample acquisition and a further 11 because of incomplete biochemical characterisation. Therefore the total study population comprised 208 patients, 140 men and 68 women. Mean age was 61 years (range 21-85); 49 (23.5\%) were smokers; 31 (15\%) were diabetic; 93 (44\%) were hypertensive; 65 (31\%) had a family history of coronary artery disease; and 73 (35\%) received treatment for hypercholesterolaemia.

\section{Discharge diagnoses}

Practice guidelines for the redefinition of AMI (ESC/ACC) and the management of patients with UA (ACC/AHA), ${ }^{21}{ }^{22}$ were used to diagnose ACS. STEMI was diagnosed if there was ST segment elevation $\geqslant 0.1 \mathrm{mV}$ in two or more contiguous leads, and NSTEMI was diagnosed if ECG was non-diagnostic and CTnT $>0.05 \mathrm{ng} / \mathrm{ml}$. All 208 patients underwent serial cTn testing at presentation and eight hours later as per institutional protocol for management of acute chest pain patients. UA was diagnosed in the presence of signs and symptoms of acute cardiac ischaemia without evidence of myocardial necrosis. Positive indications for UA were a suggestive history and clinical examination; typical ischaemic ECG changes at rest or during exercise; regional wall motion abnormality after stress echocardiography, abnormal perfusion scans or significant stenosis $(>70 \%)$ on coronary angiography. Patients were classified as nonischaemic chest pain (NICP) when (1) a reported noncardiac mechanism was confirmed as the cause of chest pain; (2) all of the following criteria were met: negative cTnT results on serial sampling (over a 6-9 hour interval), presence of normal ECGs, and absence of any current (lesions $<70 \%$ diameter reduction in any major epicardial vessel) or previous evidence of CAD; or (3) a diagnosis of ACS was objectively excluded after admission to the coronary care unit (CCU).

\section{ECG classification}

Positive ECGs were those with ST segment depression or elevation $\geqslant 0.1 \mathrm{mV}$, or $\mathrm{T}$ wave inversion $\geqslant 0.2 \mathrm{mV}$ (in $\geqslant$ two contiguous leads). ECGs showing no ST segment shifts or $\mathrm{T}$ wave changes (apart from lead III or V1) were considered negative. Equivocal or uninterpretable ECGs (that is, left bundle branch block, paced rhythm, extensive pathological Q waves, and/or persistent ST segment elevation after previous AMI) were considered to be negative in this study. Each ECG was evaluated by the ED or medical consulting physician, or both, as per routine institutional practice.

\section{Angiography and final diagnosis}

Coronary angiography was carried out on 75 patients. All angiographic images were reviewed by an experienced cardiologist blinded to the patient's clinical characteristics and biochemistry results. A positive angiogram was defined as stenosis $\geqslant 70 \%$ diameter reduction in any major epicardial vessel. Final diagnosis for this study was based on ED discharge diagnosis for patients discharged from the ED, and hospital discharge diagnosis, for patients admitted to the hospital for further investigation and management. The ED consultant or medical consultant were responsible for assigning a final diagnosis based on the history, clinical examination, serial cTnT results, and data from medical records. These included results of ECG, exercise stress testing, perfusion scans, and coronary angiography, as available. Results of all investigations were reviewed blinded to IMA results.

\section{Albumin cobalt binding test}

IMA was measured by the albumin cobalt binding test (ACB Test) on the Roche Cobas MIRA PLUS instrument. The mechanism whereby IMA represents a marker of ischaemia is based upon the fact that human serum albumin (HSA) has the ability to bind certain transition metal ions, particularly cobalt and copper, at the $\mathrm{N}$-terminus. Bar-Or has previously reported that exposure of albumin to ischaemic tissue changes the structure of HSA N-terminus such that it can no longer bind cobalt. ${ }^{23} 24$

Blood was collected for the IMA test in serum separated tubes. Specimens were frozen at $-20^{\circ} \mathrm{C}$ or colder within two hours. Frozen samples were gently vortexed after thawing. Specimens handled in this way showed no significant difference in assay results from the fresh specimens. In the ACB Test, $95 \mu \mathrm{l}$ of a patient sample and $5 \mu \mathrm{l}$ of cobalt chloride (CoII), are incubated for five minutes. During incubation, the $\mathrm{Co}(\mathrm{II})$ binds to the N-terminus of unaltered albumin in the sample; albumin for which the $\mathrm{N}$-terminus is altered as a result of ischaemic processes binds to the $\mathrm{Co}$ (II) to a far lesser extent. After incubation, $25 \mu \mathrm{l}$ of dithiothreitol (DTT) is added to the mixture. DTT forms a coloured complex with $\mathrm{Co}(\mathrm{II})$ that is not bound at the N-terminus of albumin, and this complex is measured spectrophotometrically at $500 \mathrm{~nm}$. Duplicate IMA values were obtained with the mean recorded as the result of the assay.

In our laboratory, the ACB test within-run duplicate CV\% of patient samples averaged $1.9 \%$ (range $0.0 \%-6.5 \%$ ). We applied the IMA upper limit of normal (95th centile of 111 apparently healthy people) reported by the manufacturer. IMA values $>85 \mathrm{U} / \mathrm{ml}$ were considered positive for cardiac ischaemia.

\section{Cardiac troponin T test}

Blood was collected for the cTnT test in serum separated tubes and cTnT concentrations $>0.05 \mathrm{ng} / \mathrm{ml}$ were considered positive. cTnT concentrations were measured by electrochemiluminescence assay with an Elecsys 1010 analyser (Roche Diagnostics, Lewes, Sussex, UK).

\section{Statistical analysis}

A power calculation was carried out for disproving the null hypothesis that IMA will be positive in $<70 \%$ of the ACS patients. The expected performance was to predict ACS $85 \%$ of the time. It was determined that at least 79 patients positive for ACS were required for $95 \%$ confidence in an observed sensitivity greater than $69.9 \%$.

Results of IMA, ECG, and cTnT were analysed for clinical sensitivity, specificity, positive predictive value (PPV), and negative predictive value (NPV), alone and in combination. The two and three test combinations of IMA, ECG, and CTnT were considered positive if any one of the tests was positive, and negative if all were negative. Performance estimates were compared using McNemar's test for correlated proportions and confidence intervals were calculated using the exact binomial method. Comparison of median IMA values among disease conditions was performed using the Mann-Whitney 


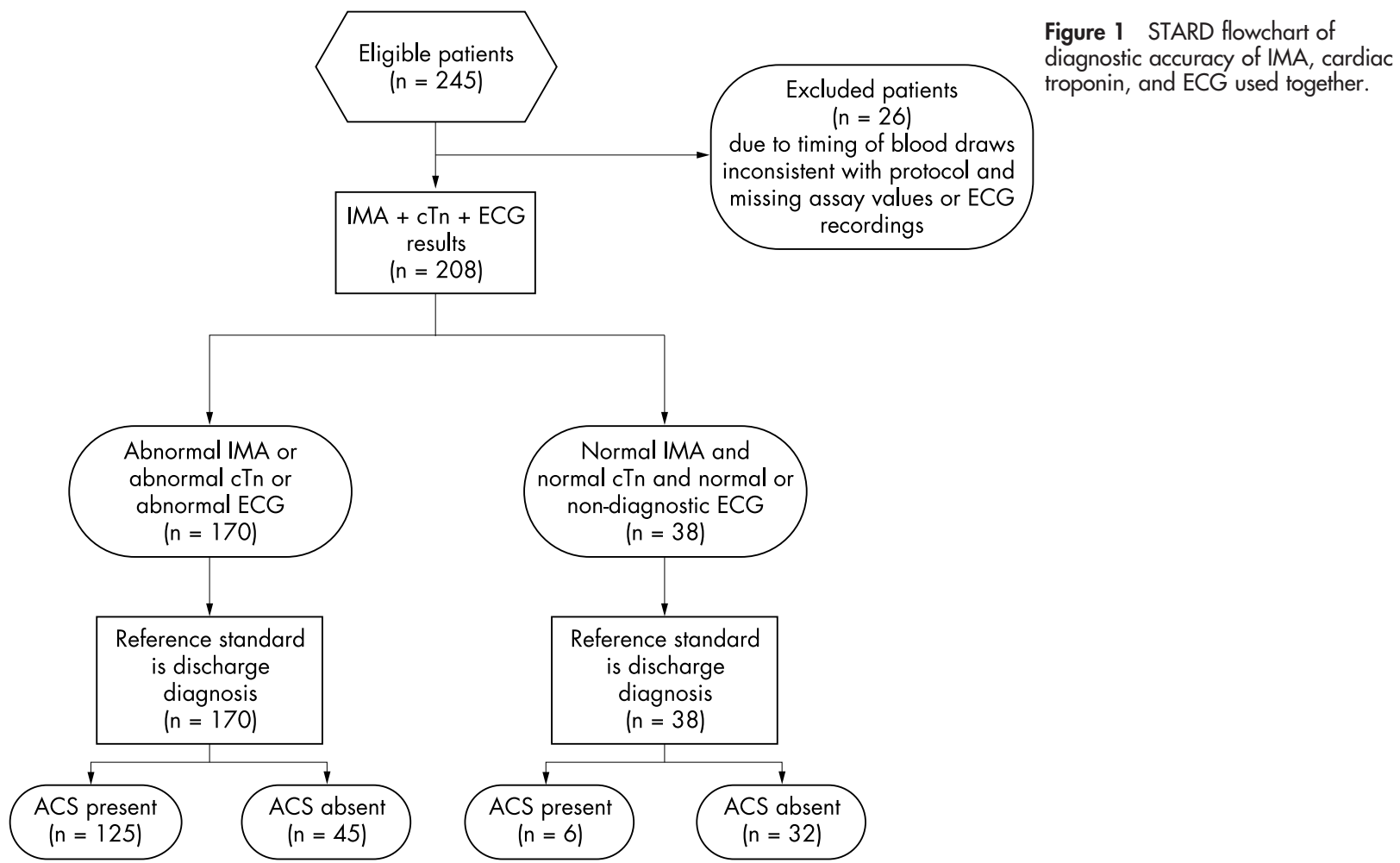

$\mathrm{U}$ test for independent samples. A two tailed p value of $<0.05$ was considered significant. All statistical tests were performed using Analyse-it Software, United Kingdom.

\section{RESULTS}

The STARD guidelines (http://www.consort-statement.org/ stardstatement.htm) were adhered to for the reporting of results from the study. Of the 208 enrolled patients, 131 were discharged with a final diagnosis of ACS and 77 with the diagnosis of NICP. Among the ACS patients, 20 had STEMI, 26 had NSTEMI, and 85 had UA (fig 1). In the UA group a clinical diagnosis was made in only 29 (34\%) cases while 56 diagnoses were based upon objective testing. Of these, 38 had $>70 \%$ stenosis on angiography and one patient with a previous history of CABG had minor disease affecting the grafts but severe disease affecting the native vessels. The remaining patient had normal coronary arteries but a history highly suggestive of coronary artery spasm. Five patients had a positive dobutamine stress echocardiogram and a further 11 had positive exercise stress tests.

Of the 77 patients with a final diagnosis of NICP, a clear non-cardiac cause of chest pain was identified in 47. Among the remaining 30, 12 patients underwent angiography and 18 had a negative exercise stress test. Among the 12 who underwent angiography, 10 had normal coronary arteries; one had $50 \%$ stenosis and a negative dobutamine stress echocardiogram and one had 50\% stenosis and no further testing. Both patients with $50 \%$ stenosis had a positive IMA result.

Median IMA values were significantly higher in patients with ACS compared with NICP $(\mathrm{p}<0.0001)$, in patients with UA compared with NICP $(\mathrm{p}<0.0001)$, and in patients with UA compared with AMI $(\mathrm{p}=0.02)$ (table 1$)$.

Performance of IMA, ECG, CTnT, used alone and in combination, for diagnosis of ACS is presented in table 2; comparison of sensitivities for statistically significant differences are illustrated in figure 2. IMA at presentation identified 107 of 131 patients $(82 \%, 95 \%$ CI 74 to 88$)$, compared with 59 of 131 (45\%, CI 36 to 54) by admission ECG and 26 of $131(20 \%$, CI 13 to 28$)$ by admission CTnT. When IMA was used with ECG, the sensitivity increased to $92 \%$ (CI 86 to 96 ) and this was similar to that of IMA used with CTnT, 90\% (CI 84 to 95). Sensitivity of the three tests combined was $95 \%$ (CI 90 to 98 ), which was significantly greater than that of IMA and cTnT combined $(p=0.02)$ and statistically equivalent to the sensitivity of IMA and ECG combined $(p=0.13)$. When ECG and cTnT were used (standard practice in our institution), only 53\% (CI 44 to 62) of patients with chest pain of ischaemic origin were identified. Logistic regression indicates that IMA is a significant addition to conventional diagnostics at ED presentation increasing the area under the receiver operator

Table 1 IMA median values by diagnosis

\begin{tabular}{lcll}
\hline & Number & Median IMA (U/ml) & $95 \%$ Cl of median \\
\hline UA or NSTEMI or STEMI & 131 & $95.4^{*}$ & 93.8 to 99.1 \\
NICP & 77 & 86.2 & 84.3 to 90.6 \\
UA & 85 & $97.3 \dagger^{*}$ & 94.2 to 100.6 \\
AMI (with or without ST elevation & 46 & 93.2 & 83.8 to 100.2 \\
NSTEMI & 26 & 94.2 & 83.8 to 101.3 \\
STEMI & 20 & 89.9 & 81.5 to 101.4 \\
\hline
\end{tabular}

*Significantly different from NICP ( $p<0.0001)$; †significantly different from AMI $(p=0.02) . \mathrm{Cl}$, confidence intervals. 


\begin{tabular}{|c|c|c|c|c|c|c|c|c|c|}
\hline \multirow[b]{2}{*}{ Test } & \multicolumn{2}{|c|}{ Sensitivity for ACS } & \multicolumn{2}{|c|}{ Specificity for ACS } & \multicolumn{2}{|c|}{ PPV for ACS } & \multicolumn{2}{|c|}{ NPV for ACS } & \multirow{2}{*}{$\begin{array}{l}\text { ROC area } \\
\text { under curve }\end{array}$} \\
\hline & $\%$ & $\mathrm{Cl}$ & $\%$ & $\mathrm{Cl}$ & $\%$ & $\mathrm{Cl}$ & $\%$ & $\mathrm{Cl}$ & \\
\hline IMA & 82 & 74 to 88 & 46 & 34 to 57 & 72 & 64 to 79 & 59 & 46 to 72 & 0.68 \\
\hline ECG & 45 & 36 to 54 & 91 & 82 to 96 & 89 & 80 to 96 & 49 & 41 to 58 & 0.68 \\
\hline CTnT & 20 & 13 to 28 & 99 & 93 to 100 & 96 & 81 to 100 & 42 & 35 to 50 & 0.64 \\
\hline ECG and CTnT & 53 & 44 to 62 & 90 & 81 to 95 & 90 & 81 to 95 & 53 & 44 to 62 & 0.74 \\
\hline IMA and cTnT & 90 & 84 to 95 & 44 & 33 to 56 & 73 & 66 to 80 & 72 & 57 to 84 & 0.77 \\
\hline IMA and ECG & 92 & 86 to 96 & 43 & 32 to 55 & 73 & 66 to 80 & 77 & 61 to 88 & 0.80 \\
\hline $\begin{array}{l}\text { IMA and ECG } \\
\text { and cTnT }\end{array}$ & 95 & 90 to 98 & 42 & 30 to 53 & 74 & 66 to 80 & 84 & 69 to 94 & 0.83 \\
\hline
\end{tabular}

characteristic (ROC) curve from 0.74, using only ECG and cTnT, to 0.83 (table 2).

The sensitivities of IMA, ECG, and CTnT, used alone and in combination, for diagnosis of UA, NSTEMI, and STEMI are presented in table 3. IMA identified more UA patients, 91\% (CI 82 to 96) compared with ECG, 32\% (CI 22 to 43 ). Identification of patients discharged with STEMI was best by ECG, 95\% (CI 75 to 100), where one patient was misdiagnosed at presentation, but developed ST elevation 12 hours later. IMA was raised in $60 \%$ of STEMI patients (CI 36 to 80 ) compared with presentation CTnT, 30\% (CI 12 to 54). Raised IMA and raised CTnT at presentation were detected in a similar number of patients with NSTEMI, 69\% (CI 48 to 85 ) and $65 \%$ (CI 44 to 83 ), respectively. Only $50 \%$ (CI 30-70) of NSTEMI patients had ischaemic ECG changes on presentation.

\section{DISCUSSION}

This study showed for the first time that the sensitivity of IMA for the diagnosis of acute ischaemic chest pain is significantly greater than that of ECG and CTnT, used alone or combined. UA with normal cTnT (cut off $=0.05 \mathrm{ng} / \mathrm{ml}$ ) accounted for $65 \%$ of the positive ACS diagnoses in this study, only $32 \%$ of which were identified at presentation by ECG, while IMA identified 91\%. Combining IMA with ECG did not statistically improve sensitivity for the detection of ischaemic UA compared with the IMA used alone. Our findings confirm and expand upon a previous report that in a human model of ischaemia induced by balloon angioplasty, IMA rises early after balloon inflation and levels returns to baseline within 6-12 hours. ${ }^{25} 26$

A biomarker of ischaemia such as IMA may improve our ability to identify ischaemic patients who are missed by current diagnostic strategies, or more confidently rule out patients who do not have ACS. More than $50 \%$ of patients presenting to the ED with chest pain are admitted to a hospital to rule in or rule out IHD. ${ }^{27}{ }^{28}$ An underlying cardiac origin of the pain is ultimately ruled out in about half of admitted patients. For efficient management in an ED, NPV may be most critical. False negatives are an undesirable outcome but true negatives are of greater importance because the accurate exclusion of ACS preserves limited and expensive resources. ${ }^{29}$ High sensitivity will drive high NPV in a low prevalence population because of the comparatively smaller number of false negatives.

Conventional ED testing in patients presenting with chest pain includes ECG and CTn. In this study, the presentation ECG combined with cTnT identified 53\% of patients with a discharge diagnosis of UA, NSTEMI, or STEMI. When IMA was added to either the ECG or CTnT measurements, or both, the sensitivity for diagnosis increased to $90 \%-95 \%$. The additive value of IMA was also confirmed by ROC curve analysis. Thus the addition of IMA to current standard tests may identify additional patients with IHD who could benefit from earlier treatment or more confidently rule out patients without IHD.

For ruling out ACS, the NPV for all three tests combined was $84 \%$, which was significantly greater than any test on its own. However, this statistic should be interpreted with caution as this particular study population has a much higher prevalence of ACS than a typical "all comers" ED chest pain population. In a population with lower prevalence, the NPV will be higher.

At present there is no gold standard for myocardial ischaemia and this is one of the main challenges faced in the testing of a marker such as IMA. In our study IMA results were compared with the final "hospital" diagnosis, which represented the "gold standard" for the purpose of the study. Final hospital diagnosis resulted from the analysis of all available clinical data-that is, the clinical history, 12-lead

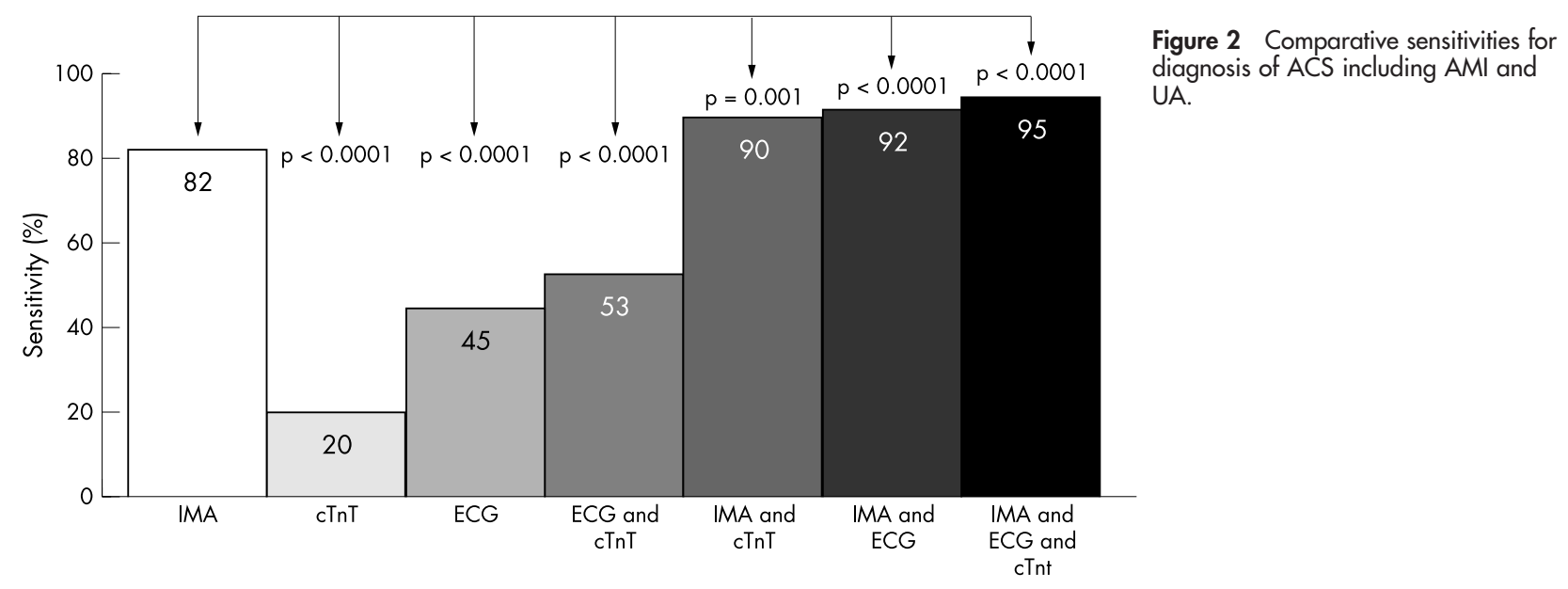


Table 3 Sensitivity of presentation diagnostic tests by diagnosis

\begin{tabular}{llll}
\hline Test & UA $(\mathbf{n}=\mathbf{8 5})$ & NSTEMI $(\mathbf{n}=\mathbf{2 6})$ & STEMI $(\mathbf{n}=\mathbf{2 0})$ \\
\hline IMA & $91(82$ to 96$)$ & $69(48$ to 86$)$ & $60(36$ to 81$)$ \\
ECG & $32(22$ to 43$)$ & $50(30$ to 70$)$ & $95(75$ to 100$)$ \\
cTnT & NA & $65(44$ to 83$)$ & $30(12$ to 54$)$ \\
ECG and cTnT & NA & $81(61$ to 93$)$ & $95(75$ to 100$)$ \\
IMA and cTnT & NA & $96(80$ to 100$)$ & $75(51$ to 91$)$ \\
IMA and ECG & $94(87$ to 98) & $81(61$ to 93$)$ & $100(83$ to 100$)$ \\
IMA and ECG and cTnT & NA & $96(80$ to 100$)$ & $100(83$ to 100$)$ \\
\hline
\end{tabular}

ECG, exercise ECG, perfusion scans, stress echocardiography, and coronary angiography.

The positive IMA results in two patients with angiographically "normal" coronary arteries $(<50 \%$ stenosis diameter) could be explained by the presence of dynamic vacoconstriction of coronary stenoses ${ }^{30}$ and coronary artery spasm $^{31}$ that may cause myocardial ischaemia in the absence of angiographically significant coronary athersclerosis.

One of the limitations of this study was the absence of an independent panel of observers to define the final diagnosis. However, we tried to mimic "real life" clinical decisions by using the hospital diagnosis. Furthermore, this study was also limited by patient selection restrictions. Patients enrolled in the study were required to have pain suggestive of myocardial ischaemia within three hours of presentation and have a clear recollection of symptom onset. These enrolment criteria precluded the recruitment of "all comers" to the ED and may have contributed, at least in part, to the high incidence of positive ACS diagnoses. Patients with comorbid cerebrovascular and peripheral vascular disease were not included as the effects of ischaemia in other vascular territories has not yet been fully characterised. In this selected population our aim was to objectively assess the ability of IMA to detect ACS without the presence of any confounding clinical conditions that may affect our ability to interpret results. It is possible that increases in IMA could, in theory be observed during ischaemia affecting any organ. Increased concentrations of IMA have been demonstrated 24-48 hours after endurance exercise and it has been hypothesised this is related to delayed gastrointestinal or skeletal muscle ischaemia. ${ }^{32}$

This study shows that IMA has high sensitivity for prediction of a discharge diagnosis of ACS, but comparatively low specificity (in contrast with presentation troponin, which has high specificity but very low sensitivity). One of the reasons for this may well be that IMA is detecting ischaemia that is subclinical and beyond the ability of conventional diagnostic methods to identify. In addition, we used stringent criteria for a positive angiogram (that is, $\geqslant 70 \%$ stenosis). Therefore a number of patients with lesser stenoses but capable of causing myocardial ischaemia were classified as NICP.

In this study, there was a significant, albeit comparatively small difference in IMA values when patients with NICP and those with UA were compared. This may be accounted for by selection bias as the patients included in the study were highly likely to have ACS.

The fact that only $60 \%$ of STEMI patients had high IMA levels is puzzling as is our finding that IMA was higher in patients with UA without myocardial necrosis compared with those with AMI. It may be speculated that total acute occlusion of the culprit artery causes acute tissue necrosis that prevails over ischaemia limiting access of the modified HSA to the systemic circulation. In addition, with myocardial necrosis less albumin will be exposed to circulating free radicals resulting in lesser IMA production. Because of the difficulty of pinpointing the exact time of onset of an ischaemic event, there is always the possibility that IMA was initially raised but had already decreased below the diagnostic cut off at the time of the blood draw. These intriguing findings do not detract from the fact that IMA is a sensitive marker of ischaemia (rather than necrosis). From a practical clinical perspective, STEMI is often an unequivocal diagnosis indicating the urgent need for thrombolytic therapy or coronary intervention and IMA testing may be less clinically relevant in this setting.

\section{CONCLUSIONS}

We have shown that IMA has potential as a diagnostic biomarker for UA. Significantly more UA patients were recognised at presentation by IMA than by ECG and the combination of IMA, ECG, and CTnT would have permitted earlier discharge of more patients with NICP. If the present results are confirmed by additional studies, the use of IMA may improve current diagnostic strategies for acute chest pain patients.

\section{ACKNOWLEDGEMENTS}

The authors thank Elizabeth Cornu for management of the clinical database and Deborah Morris for invaluable statistical analysis and comments.

\section{Authors' affiliations}

M K Sinha, D Roy, J-C Kaski, Coronary Artery Disease Research Unit, St George's Hospital Medical School, London, UK

D C Gaze, P O Collinson, Chemical Pathology, St George's Hospital, London, UK

Funding: MKS is the recipient of an unrestricted educational grant from Ischemia Technologies and POC is on the scientific advisory board of Ischemia Technologies.

Conflicts of interest: none declared.

\section{REFERENCES}

1 Burt CW. Summary statistics for acute cardiac ischemia and chest pain visits to United States emergency departments, 1995-1996. Am J Emerg Med 1999; 17:552-9.

2 Pope JH, Aufderheide TP, et al. Missed diagnosis of acute cardiac ischemia in the emergency department. New Engl J Med 2000;32:1163-70.

3 Fuster V, Lewis A. Conner Memorial Lecture. Mechanisms leading to myocardial infarction: insights from studies of vascular biology. Circulation 1994;90:2126-46.

4 Fuster V, Badimon L, Badimon JJ, et al. The pathogenesis of coronary artery disease and the acute coronary syndromes (first of two parts). N Engl J Med 1992;326:242-50

5 Fuster V, Badimon L, Badimon JJ, et al. The pathogenesis of coronary artery disease and the acute coronary syndromes (second of two parts). N Engl J Med 1992;326:310-18.

6 Ambrose JA. Plaque disruption and the acute coronary syndromes of unstable angina and acute myocardial infarction: If the substrate is similar, why is the clinical presentation different? J Am Coll Cardiol 1992;19:1653-8.

7 Falk E. Unstable angina with fatal outcome: dynamic coronary thrombosis leading to infarction and/or sudden death: autopsy evidence of recurrent mural thrombosis with peripheral embolization culminating in total vascular occlusion. Circulation 1985;71:699-708.

8 Tatum JL, Jesse RL, Kontos MC, et al. Comprehensive strategy for the evaluation and triage of the chest pain patient. Ann Emerg Med 1997;29:1 16-25. 
9 Jesse RL, Kontos MC. Evaluation of chest pain in the emergency department. Curr Probl Cardiol 1997;22:149-236.

10 Sgarbossa EB, Birnbaum Y, Parrillo JE. Electrocardiographic diagnosis of acute myocardial infarction: current concepts for the clinician. Am Heart J 2001;141:507-17.

11 Lieberman AN, Weiss JL, Jugdutt BI, et al. Two-dimensional echocardiography and infarct size: relationship of regional wall motion and thickening to the extent of myocardial infarction in the dog. Circulation $1981 ; 63: 739-46$

12 Bilodeau L, Theroux P, Gregoire J, et al. Technetium- $99 \mathrm{~m}$ sestamibi tomography in patients with spontaneous chest pain: correlations with clinical, electrocardiographic and angiographic findings. J Am Coll Cardiol 1991;18:1684-91.

13 Selker HP, Zalenski RJ. Emergency diagnostic tests for cardiac ischaemia: a report from the National Heart Attack Alert Program (NHAAP). Malden, MA: Blackwell Science, 1997.

14 Kontos MC, McQueen RH, et al. Can myocardial infarction be rapidly identified in emergency department patients who have left bundle branch block? Ann Emerg Med $2001 ; 37: 431-8$.

15 Peels C, Visser CA, Funkelkupper AJ, et al. Usefulness of two-dimensional echocardiography for immediate detection of myocardial ischemia in the emergency room. Am J Cardiol 1990;65:687-91.

16 Iskandrian AS, Heo J, Kang B, et al. Effect of exercise level on the ability of thallium 201 tomographic imaging in detecting coronary artery disease:analysis of 461 patients. J Am Coll Cardiol 1989;14:1477-86

17 Wu AHB. Cardiac markers. Totowa, NJ: Humana Press, 1998.

18 Christenson RL, Duh SH, Sanhai WR, et al. Characteristics of an albumin cobalt binding test for assessment of acute coronary syndrome patients: a multicenter study. Clin Chem 2001;47:464-70.

19 Wu AB, Morris DL, Fletcher DR, et al. Analysis of the albumin cobalt binding (ACB) test as an adjunct to cardiac troponin I for the early detection of acute myocardial infarction. Cardiovasc Toxicol 2001;1:147-51.

20 Bhagavan NV, Lai EM, Rios RA, et al. Evaluation of human serum albumin cobalt binding assay for the assessment of myocardial ischemia and myocardial infarction. Clin Chem 2003;49:581-5.

21 The Joint European Society of Cardiology and American College of Cardiology Committee Myocardial Infarction. Redefined-a consensus document of the Joint European Society of Cardiology/American College of Cardiology Committee for the redefinition of myocardial infarction. J Am Coll Cardiol 2000;36:959-69.

22 Braunwald E, Antman EM, Beasley JW, et al. ACC/AHA guidelines for the management of patients with unstable angina and non-ST segment elevation myocardial infarction. J Am Coll Cardiol 2000;36:970-1062.

23 Bar-Or D, Curtis G, Rao N, et al. Characterization of the Co2+ and $\mathrm{Ni2}+$ binding amino-acid residues of the $\mathrm{N}$-terminus of human albumin. Eur J Biochem $2001 ; 268: 42-7$.

24 Bar-Or D, Lau E, Winkler JV. A novel assay for cobalt-albumin binding and its potential as a marker for myocardial ischaemia-a preliminary report. $J$ Emerg Med 2000;19:31 1-15.

25 Bar-Or D, Winkler J, VanBenthuysen K, et al. Reduced cobalt binding of human albumin with transient myocardial ischaemia following elective percutaneous transluminal coronary angioplasty compared to $C K-M B$, myoglobin and troponin I. Am Heart J 2001;141:985-91.

26 Sinha MK, Gaze DC, Collinson PO, et al. Ischemia modified albumin is a sensitive marker of ischemia following percutaneous coronary intervention. Circulation 2003;107:2403-5.

27 Goldman L, Weinberg M, Weisberg M, et al. A computer derived protocol to aid in the diagnosis of ER patients with acute chest pain. N Engl J Med 1982;307:588-96

28 Selker HP, Beshansky JR, Griffith JL, et al. Use of the acute cardiac ischemia time insensitive predictive instrument (ACl-TIPI) to assist with triage of patients with chest pain or other symptoms suggestive of acute cardiac ischemia. A multicentre, controlled clinical trial. Ann Intern Med 1998;129:845-55.

29 Einstein AJ, Bodiam CA, Gill J. The relationships among performance measures in selection of diagnostic tests. Arch Pathol Lab Med 1997;121:110-17.

30 Kaski JC, Tousoulis D, Haider AW, et al. Reactivity of eccentric and concentric coronary stenoses in patients with chronic stable angina. J Am Coll Cardiol $1991 ; 17: 627-33$

31 Maseri A, Davies G, Hackett D, et al. Coronary artery spasm and vasoconstriction. The case for a distinction. Circulation 1990;81:1983-91.

32 Apple FS, Quist HE, Otto AP, et al. Release characteristics of cardiac biomarkers and ischemia modified albumin as measured by the albumin cobalt binding test after a marathon race. Clin Chem 2002;48:1097-100. 